\title{
An often unrecognized cause of thunderclap headache: reversible cerebral vasoconstriction syndrome
}

\author{
K. Koopman $\cdot$ L. K. Teune $\cdot$ M. ter Laan \\ M. Uyttenboogaart · P. C. Vroomen · J. De Keyser · \\ G. J. Luijckx
}

Received: 23 June 2008/Accepted: 31 August 2008/Published online: 23 September 2008

(C) The Author(s) 2008. This article is published with open access at Springerlink.com

\begin{abstract}
Thunderclap headache (TCH) can have several causes of which subarachnoid hemorrhage (SAH) is most common and well known. A rare cause of $\mathrm{TCH}$ is the reversible cerebral vasoconstriction syndrome (RCVS) which is characterized by a reversible segmental vasoconstriction of the intracranial vessels. We describe two patients with TCH due to RCVS and the probable precipitating factor, namely, cannabis and an anti-migraine drug. In RCVS, cerebrospinal fluid examination is (near) normal, in contrast to SAH and (primary) cerebral vasculitis. Brain MRI may be normal or shows infarction. MRA can demonstrate vasoconstriction of the great arteries, but a normal MRA does not rule out the diagnosis. Caliber changes on cerebral angiography cannot adequately differentiate between RCVS and vasculitis. Calcium-channel antagonists may be a good therapy and repeated transcranial Doppler ultrasonography can be a reliable non-invasive investigation to monitor the effect of treatment and demonstrate reversibility of the vasoconstriction.
\end{abstract}

Keywords Thunderclap headache ·

Reversible cerebral vasoconstriction syndrome .

Vasospasm

K. Koopman $(\varangle) \cdot$ L. K. Teune · M. Uyttenboogaart ·

P. C. Vroomen - J. De Keyser - G. J. Luijckx

Department of Neurology, University Medical Center

Groningen, University of Groningen, Hanzeplein 1,

9700 RB Groningen, The Netherlands

e-mail: k.koopman@neuro.umcg.nl

M. ter Laan

Department of Neurosurgery, University Medical Center

Groningen, University of Groningen, Groningen,

The Netherlands

\section{Introduction}

Thunderclap headache (TCH) is often associated with a subarachnoid hemorrhage (SAH). However, TCH can also be the first sign of several other neurological conditions, some of which are less well known [1]. The term primary $\mathrm{TCH}$ can be used when all other potential conditions have been eliminated by diagnostic testing. A rare cause of TCH is the reversible cerebral vasoconstriction syndrome (RCVS) [2]. This syndrome is characterized by a reversible segmental vasoconstriction of the intracranial blood vessels [2-5]. It is important to recognize this syndrome and distinguish RCVS from other conditions such as primary angiitis of the central nervous system (PACNS), to choose proper treatment $[3,4,6]$. Here, we present two patients with TCH due to RCVS and discuss the clinical features of this often unrecognized syndrome.

\section{Case 1}

A 46-year-old man with a history of radical extirpated melanoma was transferred to our hospital under the suspicion of cerebral vasculitis. One week before, he presented with spontaneous acute severe headache, followed a few days later by progressive confusion and a mild left-sided hemiparesis. Magnetic imaging resonance (MRI) of the brain showed ischemic lesions bilateral occipital and right parietal. MR angiography (MRA) showed no abnormality; however, on second review the examination was without gadolinium and of poor quality and therefore not reliable. Patient did not use any medication, smoked 20 cigarettes and 3-4 times cannabis a day. The admission blood pressure was 184/89 and the following day 154/90. On neurological examination, he appeared confused and 
scored 15 of 30 items on the mini-mental state examination. There was a hemiparesis MRC grade 4 on the left side. Ancillary laboratory investigations in serum (including blood count, serum electrolytes, liver, renal and thyroid function tests, erythrocyte sedimentation rate, C-reactive protein level, antinuclear and antineutrophil cytoplasmic antibody tests, complement $\mathrm{C} 1 \mathrm{q}, \mathrm{C} 3$ and $\mathrm{C} 4$, lupus anticoagulant, rheumatoid factor) and cerebrospinal fluid (CSF) showed no abnormalities. Selective catheter cerebral angiography showed multiple caliber changes in the intracranial blood vessels (Fig. 1). Transcranial Doppler ultrasonography (TCD) examination showed increased intracranial blood flow velocities among others of medial cerebral artery $(154 \mathrm{~cm} / \mathrm{s})$, and anterior cerebral artery $(168 \mathrm{~cm} / \mathrm{s})$. Because of the acute presentation with $\mathrm{TCH}$, normal CSF examination and the findings on TCD the diagnosis RCVS was established. Calcium-channel antagonist nimodipine $60 \mathrm{mg}$ six times daily was started. Few days after treatment was started, the clinical condition improved. A follow-up TCD showed a normalization of blood flow velocities (medial cerebral artery: $94 \mathrm{~cm} / \mathrm{s}$ and anterior cerebral artery: $90 \mathrm{~cm} / \mathrm{s}$ ). The follow-up MRI showed reduction in size of the hyperintense lesions. The patient was transferred to a rehabilitation center and discharged home after 3 weeks under good clinical condition.

\section{Case 2}

A 48-year-old woman presented at the emergency department with spontaneous acute severe headache. She had a history of migraine, but did not recognize this type of headache as her migraine headache. She frequently used sumatriptan and 1 week before admission, because of severe attacks, she had used sumatriptan daily with temporally good response. On examination, the blood pressure was 140/88 and neurological examination was normal. SAH was ruled out by CT scan and CSF examination was completely normal. Following day, she became confused with behavioral changes. Brain MRI/MRA revealed no abnormalities. TCD showed increased blood flow velocities over the medial cerebral artery $(109 \mathrm{~cm} / \mathrm{s})$ and anterior cerebral artery $(108 \mathrm{~cm} / \mathrm{s})$. Under the suspicion of RCVS, calcium antagonist verapamil $120 \mathrm{mg}$ twice daily was started. Within a day, the patient improved clinically and TCD after 4 days showed normal blood flow velocities of medial cerebral artery $(80 \mathrm{~cm} / \mathrm{s})$ and anterior cerebral artery $(66 \mathrm{~cm} / \mathrm{s})$. She was discharged at home without any complaints and was strongly advised not resume any triptans.

\section{Discussion}

These cases highlight the recognition of RCVS as a cause of TCH. It is important to realize that TCH is a symptom rather than a diagnosis and can be caused by several acute (neurological) conditions as cerebral venous thrombosis, ischemic stroke, dissection, acute hypertension, vasospasm and infection [1].

The RCVS was first described by Call et al. in 1988 [2] and is characterized by a reversible segmental vasoconstriction of the intracranial vessels. It is most common in women aged $20-50$ years. The striking presenting feature of RCVS is TCH, followed immediately, or after a symptom free interval, by seizures, neurological deficits or encephalopathy [2-5]. RCVS can occur spontaneously or in association with pregnancy, migraine, sexual activity, certain prescribed or illicit drugs (selective serotonin reuptake inhibitors, triptans, cannabis, cocaine and ecstasy) [3-5, 7-10]. Concerning the patients in our case, the frequent use of cannabis in the first patient and sumatriptan in the second patient were probably the precipitating factors.
Fig. 1 Cerebral angiography: multiple caliber changes in the intracranial vessels

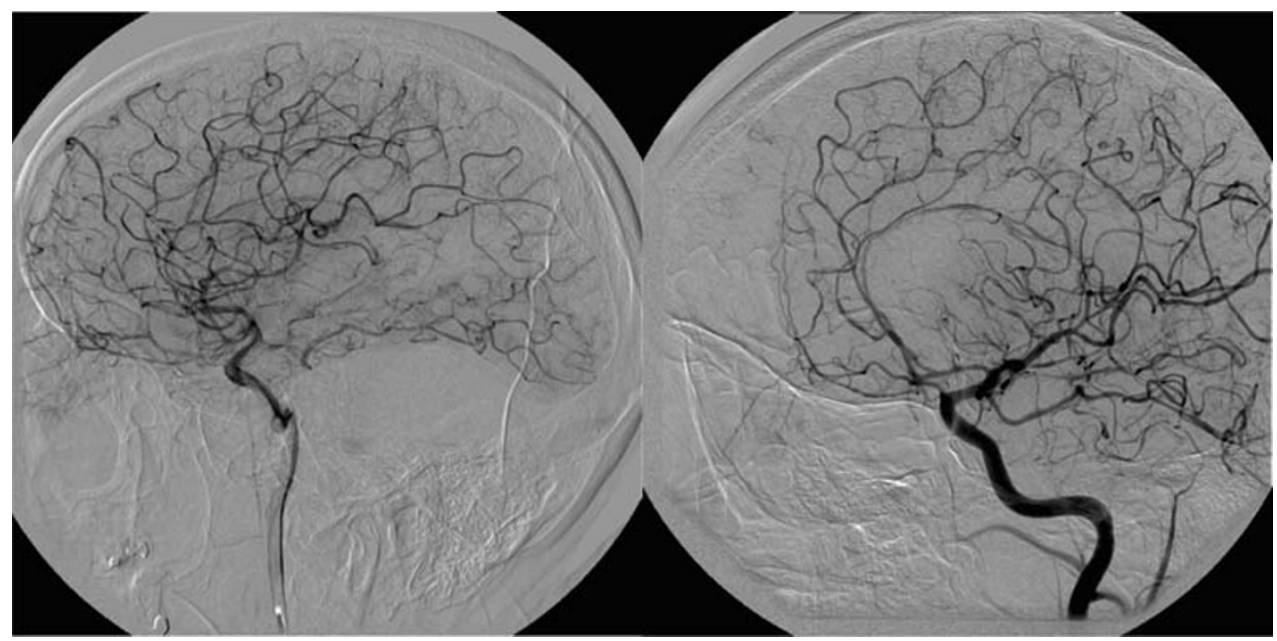


MRI findings in RCVS can be normal or showing ischemic lesions, especially in posterior circulation and watershed areas. CSF is normal in $80-90 \%$ of the patients, as it was in our patients [3-6].

MR angiography can demonstrate vasoconstriction in the proximal parts of the great cerebral arteries. However, a normal MRA examination does not rule out the diagnosis. In a series of 44 RCVS patients, 6 had a normal MRA while on cerebral angiography there were signs of vasoconstriction [7]. In the first patient MRA was inconclusive but cerebral infarction was seen on MRI and cerebral angiography showed multiple alterations. MRA in the second patient did not show vasospasm; the reason might be that this patient had only mild vasospasm.

Cerebral angiography can show multiple alterations of the cerebral blood vessel caliber. These findings are often associated with cerebral vasculitis or PACNS but these patients present in general with gradually progressive neurological symptoms, encephalopathy and CSF is abnormal in $80-90 \%$ [1, 6, 11].

TCD is not specific for the initial diagnosis of RCVS, but (repeated) TCD can be a reliable non-invasive investigation to monitor vasospasm. Normalization of cerebral blood flow velocities is often seen within 12 weeks $[5,12]$. In our patients there was also normalization of cerebral blood flow velocities.

The exact pathophysiology underlying the reversible vasoconstriction is unknown. The International Classification of Headache Disorders-II classified RCVS as a secondary headache disorder, in contrast to primary $\mathrm{TCH}$. Alternatively, Chen et al. suggested that primary $\mathrm{TCH}$, or TCH elicited by, e.g., physical stress and RCVS are different spectra of the same disorder. Their study showed vasospasm in $39 \%$ of the patients with recurrent primary TCH [13]. This suggests that RCVS may have a higher incidence than reported earlier.

The sympathetic nervous system may play a role in the pathogenesis of $\mathrm{TCH}$. This is suggested by the fact that TCH has been associated with factors elevating sympathetic tone, such as adrenergics and physical stress [14]. Furthermore, proximal parts of the intracranial vessels are strongly innervated by sympathetic afferents, which modulate vascular tone. Vasoconstriction in RCVS is most common in these large- and medium-sized vessels $[1,4]$.

In RCVS, there is no established therapy. A few small observational studies and case reports described a good effect of calcium-channel antagonists in the treatment of the vasospasm of intracranial vessels [3, 15]. In summary, TCH is a symptom which can be caused by several (neurological) conditions. A normal brain CT scan and CSF examination can exclude a SAH but are not sufficient to establish "primary TCH". A rare cause of TCH is RCVS.
The exact cause of RCVS is unknown but among others associated with substance and medication use. Sometimes, this syndrome can be difficult to distinguish from PACNS on cerebral angiography and can be a pitfall. The acute presentation with TCH and the normal CSF examination are in favor of RCVS. Calcium-channel antagonists like nimodipine may be a good therapy and repeated TCD can be a reliable non-invasive investigation to monitor the effect of treatment and demonstrate the reversibility.

\section{Conflict of interest None.}

Open Access This article is distributed under the terms of the Creative Commons Attribution Noncommercial License which permits any noncommercial use, distribution, and reproduction in any medium, provided the original author(s) and source are credited.

\section{References}

1. Schwedt TJ, Matharu MS, Dodick DW (2006) Thunderclap headache. Lancet Neurol 5:621-631

2. Call GK, Fleming MC, Sealfon S et al (1988) Reversible cerebral segmental vasoconstriction. Stroke 19:1159-1170

3. Bernstein RA (2006) Reversible cerebral vasoconstriction syndromes. Curr treat Options Cardiovasc Med 8:229-234

4. Singhal AB (2004) Cerebral vasoconstriction syndromes. Top Stroke Rehabil 11:1-6

5. Calabrese LH, Dodick DW, Schwedt TJ et al (2007) Narrative review: reversible cerebral vasoconstriction syndromes. Ann Intern Med 146:34-44

6. Koopman K, Uyttenboogaart M, Luijckx GJ et al (2007) Pitfalls in the diagnosis of reversible cerebral vasoconstriction syndrome and primary angiitis of the central nervous system. Eur J Neurol 14:1085-1087

7. Ducros A, Boukobza M, Porcher R et al (2007) The clinical and radiological spectrum of reversible cerebral vasoconstriction syndrome: a prospective series of 67 patients. Brain 130:30913101

8. Noskin O, Jafarimojarrad E, Libman RB et al (2006) Diffuse cerebral vasoconstriction (Call-Fleming syndrome) and stroke associated with antidepressants. Neurology 67:159-160

9. Moussouttas M (2004) Cannabis use and cerebrovascular disease. Neurologist 10:47-53

10. Valenca MM, Valenca LP, Bordini CA et al (2004) Cerebral vasospasm and headache during sexual intercourse and masturbatory orgasms. Headache 44:244-248

11. Calabrese LH (1995) Vasculitis of the central nervous system. Rheum Dis Clin North Am 21:1059-1076

12. Chen SP, Fuh JL, Chang FC et al (2008) Transcranial color Doppler study for reversible cerebral vasoconstriction syndromes. Ann Neurol 63:751-757

13. Chen SP, Fuh JL, Lirng JF et al (2006) Recurrent primary thunderclap headache and benign CNS angiopathy: spectra of the same disorder? Neurology 67:2164-2169

14. Lu SR, Liao YC, Fuh JL et al (2004) Nimodipine for treatment of primary thunderclap headache. Neurology 62:1414-1416

15. Dodick DW (2003) Reversible segmental cerebral vasoconstriction (Call-Fleming syndrome): the role of calcium antagonists. Cephalalgia 23:163-165 